\title{
Cerebral vasomotor reactivity in epilepsy patients
}

\author{
Vijay K. Sharma $\cdot$ Hock Luen Teoh $\cdot$ \\ Bernard P. L. Chan
}

Received: 6 February 2010/Revised: 4 March 2010/Accepted: 10 March 2010/Published online: 26 March 2010

(c) Springer-Verlag 2010

Dear Sirs,

We read with interest the research paper by Bek et al. [1] about an increased vasomotor reactivity in patients with idiopathic generalized epilepsy. The paper is thought provoking and generates some important issues.

The brain maintains a constant cerebral blood flow over a wide range of blood pressure by varying the diameter of the intracranial arterioles, commonly known as vasomotor reactivity (VMR) [2]. The commonest agent to evaluate VMR is carbon dioxide $\left(\mathrm{CO}_{2}\right)$. Markus et al. [3] described the breath-holding index (BHI) as a measure of VMR. BHI is calculated from simple measurements of the flow velocities in the middle cerebral artery in response to hypercapnoea during voluntary breath-holding.

We agree with Bek et al. [1] that BHI has been validated in a variety of medical disorders. However, BHI may not be reliable in patients who are unable to hold their breath for a sufficient time. Furthermore, vasodilatory responses, reflected by increments in flow velocities do not bear a linear relationship to the breath-holding time and repeated measurements may vary widely, even in the same subject. Accordingly, wide inter-subject and intra-subject variations were observed by Bek et al. [1], both in normal controls as well as epilepsy patients. Hence, absolute BHI values cannot be used for comparison to grade VMR among the tests. While a reduced BHI represents an insufficient vasodilatory reserve (also VMR), all patients with BHI values higher than the normal threshold should be labeled as having normal VMR. We are unaware of any phenomenon called exaggerated VMR. One possible method that could ensure the reliability of individual BHI values in a single subject is by performing the test under strictly controlled conditions and monitoring the end-tidal carbon-dioxide levels.

Finally, since BHI is primarily driven by carbon-dioxide levels, an increased VMR in epilepsy patients might not represent an adaptive mechanism to protect the brain from hypoxic challenges during the seizure. We hypothesize that apnoea during the 'tonic phase' could be an adaptive measure to increase blood flow to the brain and prepare the neurons for a prolonged and enhanced metabolic and electrical activity during the seizure.

\section{References}

1. Bek S, Kaşikçi T, Koç G, Genç G, Demirkaya S, Gökçil Z, Odabaşi Z (2009) Cerebral vasomotor reactivity in epilepsy patients. J Neurol. doi: 10.1007/s00415-009-5428-4

2. Harper AM, Glass HI (1965) Effect of alteration in the arterial carbon dioxide tension on the blood flow through the cerebral cortex at normal and low arterial blood pressures. JNNP 28:449452

3. Markus HS, Harrison MJ (1992) Estimation of cerebrovascular reactivity using transcranial Doppler, including the use of breathholding as the vasodilatory stimulus. Stroke 23:668-673
V. K. Sharma $(\bowtie) \cdot$ H. L. Teoh · B. P. L. Chan

Division of Neurology, Department of Medicine, National University Hospital, Singapore 119074, Singapore e-mail: drvijay@singnet.com.sg 